\title{
Interaction between DBI-essence and other Dark Energies
}

\author{
Surajit Chattopadhyay ${ }^{1 *}$ and Ujjal Debnath ${ }^{2 \dagger}$ \\ ${ }^{1}$ Department of Computer Application, Pailan College of Management \\ and Technology, Bengal Pailan Park, Kolkata-700 104, India. \\ ${ }^{2}$ Department of Mathematics, Bengal Engineering and Science University, Shibpur, Howrah-711 103, India.
}

(Dated: October 26, 2018)

\begin{abstract}
The present work considers interaction between DBI-essence and other candidates of dark energies like modified Chaplygin gas, hessence, tachyonic field, and new agegraphic dark energy. The potentials of the fields have been reconstructed under interaction and their evolutions have been viewed against cosmic time $t$ and scalar field $\phi$. Equation of state parameters have also been obtained. The nature of potentials and the equation of state parameters of the dark energies have been found graphically in presence of interaction (both small and large interaction).
\end{abstract}

PACS numbers:

\section{INTRODUCTION}

Plethora of literatures have discussed the fact that the universe is not only expanding, but it is accelerating also $[1,2]$. Since the discovery of the fact that presently there is an accelerating expansion of the universe; different suggestions have been made in order to explain the accelerated expansion. Among the various suggestions, one is the hypothesis of dark energy, which is a fluid of negative pressure representing about $70 \%$ of the total energy of the universe [3]. The dark energy problem continues to be a sticking point for theoretical physicists. The simplest solution to this problem is to postulate the existence of a vacuum energy or cosmological constant $\Lambda$, which agrees with all the current observational bounds [4]. The cosmological constant corresponds to a fluid with a constant equation of state $\omega=-1$. Now, the observations which constrain the value of $\omega$ today to be close to that of the cosmological constant actually say relatively little about the time evolution of $\omega$, and so we can broaden our horizons and consider a situation in which the equation of state of dark energy changes with time, such as in inflationary cosmology. In 1998, the accelerated expansion of the universe was pointed out by two groups from the observations of Type Ia Supernova (SNe Ia). Unified models of the two main components of the universe, dark energy and dark matter, represent an interesting option for explaining the substantial evidence of the current acceleration of the universe. Following the tradition of trying to find an interconnection between the world of Particle Physics and Cosmology, it is customary to try and view unified dark energy models as scalar field scenarios with much steeper potentials than in the standard weakly coupled slow roll inflation model [5].

There have been many works aimed at connecting the string theory with inflation. While doing so, various ideas in string theory based on the concept of branes have proved themselves fruitful. Scenarios where the inflation is interpreted as the distance between two branes moving in the extra dimension along a warped throat have given rise to many interesting studies [6]. One area which has been well explored in recent years, is inflation driven by the open string sector through dynamical Dp-branes. This is the so-called DBI (Dirac-Born-Infield) inflation [7], which lies in a special class of K-inflation models. It was originally thought that such models yielded large levels of non-Gaussian perturbations which could be used as a falsifiable signature of string theory $[3,7]$. However subsequent work has shown that this is may not be the case, and that the simplest DBI models are essentially indistinguishable from standard field theoretic slow roll models [8]. Martin and Yamaguchi [3] introduced a scalar field model where the kinetic term has a DBI form and considered that the dark energy scalar field is a DBI scalar field, for which the action of the field can be written as

$$
S_{D B I}=-\int d^{4} x a^{3}(t)\left[T(\phi) \sqrt{1-\frac{\dot{\phi}^{2}}{T(\phi)}}+V(\phi)-T(\phi)\right]
$$

\footnotetext{
*surajit_2008@yahoo.co.in

†ujjaldebnath@yahoo.com, ujjal@iucaa.ernet.in
} 
where $T(\phi)$ is the tension and $V(\phi)$ is the potential.

To obtain a suitable evolution of the Universe an interaction is often assumed such that the decay rate should be proportional to the present value of the Hubble parameter for good fit to the expansion history of the Universe as determined by the Supernovae and CMB data [9]. These kind of models describe an energy flow between the components so that no components are conserved separately. There are several work on the interaction between dark energy (tachyon or phantom) and dark matter [10], where phenomenologically introduced different forms of interaction term.

In the present work, we would consider the interactions between different candidates of dark energy and DBI-essence model. As other models of dark energy we would consider Chaplygin gas, hessence and tachyonic field. Organization of the rest of the paper is as follows. In section II, we would consider the basic equations. In section III, we would consider the interaction between DBI-essence and Chaplygin gas and in section IV, we would consider the interaction between DBI-essence and hessence. The interaction between DBI-essence and tachyonic field would be considered in section V. In section VI, the interaction between DBI-essence and new agegraphic dark energy would be considered. The results would be discussed in section VII.

\section{BASIC EQUATIONS}

We consider a spatially flat isotropic and homogeneous universe in the FRW model whose metric is given by

$$
d s^{2}=d t^{2}-a^{2}(t)\left[d r^{2}+r^{2}\left(d \theta^{2}+\sin ^{2} d \phi^{2}\right)\right]
$$

where, $a(t)$ is the scale factor. The Einstein field equations are given by (choosing $8 \pi G=c=1)$

$$
\begin{gathered}
3 \frac{\dot{a}^{2}}{a^{2}}=\rho \\
6 \frac{\ddot{a}}{a}=-(\rho+3 p)
\end{gathered}
$$

The energy conservation equation is given by

$$
\dot{\rho}+3 \frac{\dot{a}}{a}(\rho+p)=0
$$

If we consider a model consisting of two component mixture, the an interaction term needs to be introduced. In a two-component model, we replace $\rho$ and $p$ of equations $(3),(4)$ and (5) by $\rho_{\text {total }}$ and $p_{\text {total }}$ where

$$
\rho_{\text {total }}=\rho_{D}+\rho_{X}
$$

$$
p_{\text {total }}=p_{D}+p_{X}
$$

where $\rho_{D}$ and $p_{D}$ denote the density and pressure for the DBI-essence. The terms $\rho_{X}$ and $p_{X}$ denote the density and pressure corresponding to the other dark energies. Therefore, equations (3), (4) and (5) get modified to

$$
\begin{gathered}
3 \frac{\dot{a}^{2}}{a^{2}}=\left(\rho_{D}+\rho_{X}\right) \\
6 \frac{\ddot{a}}{a}=-\left[\left(\rho_{D}+\rho_{X}\right)+3\left(p_{D}+p_{X}\right)\right]
\end{gathered}
$$




$$
\left(\dot{\rho}_{D}+\dot{\rho}_{X}\right)+3 \frac{\dot{a}}{a}\left[\left(\rho_{D}+\rho_{X}\right)+\left(p_{D}+p_{X}\right)\right]=0
$$

Assuming gravity to obey four-dimensional general relativity with a standard Einstein-Hilbert Lagrangian, the density and pressure for DBI-essence are read as [7]

$$
\begin{gathered}
\rho_{D}=(\gamma-1) T\left(\phi_{D}\right)+V_{D}\left(\phi_{D}\right) \\
p_{D}=\left(\frac{\gamma-1}{\gamma}\right) T\left(\phi_{D}\right)-V_{D}\left(\phi_{D}\right)
\end{gathered}
$$

where, $T\left(\phi_{D}\right)$ is the tension, $V_{D}\left(\phi_{D}\right)$ is the potential, $\phi_{D}$ denotes the scalar field for DBI-essence and the quantity $\gamma$ is reminiscent of the usual Lorentz factor given by

$$
\gamma=\frac{1}{\sqrt{1-\frac{\dot{\phi}_{D}^{2}}{T\left(\phi_{D}\right)}}}
$$

Since we are considering two-component model, we consider the interaction term $3 H \delta \rho_{X}$ and using (10) we can write the conservation equations as

$$
\begin{gathered}
\dot{\rho}_{D}+3 H\left(\rho_{D}+p_{D}\right)=3 H \delta \rho_{X} \\
\dot{\rho}_{X}+3 H\left(\rho_{X}+p_{X}\right)=-3 H \delta \rho_{X}
\end{gathered}
$$

where, $H=\frac{\dot{a}}{a}$ is the Hubble parameter, $\delta$ is the interaction parameter and rest of the symbols are as explained earlier.

\section{INTERACTION WITH CHAPLYGIN GAS}

Recently the so-called Chaplygin gas, also dubbed quartessence, was suggested as a candidate of a unified model of dark energy and dark matter [11]. Pure Chaplygin gas is a candidate for dark energy, which obeys an exotic equation of state

$$
p_{c h}=-\frac{A}{\rho_{c h}}
$$

where, where $p_{c h}$ and $\rho_{c h}$ are the pressure and energy density respectively and $A$ is a positive constant. This equation leads to a density evolution in the form

$$
\rho_{c h}=\sqrt{A+\frac{B}{a^{6}}}
$$

where, $B$ is an integration constant. The model unifies both dark energy and dark matter. The reason is that, from (17), the Chaplygin gas behaves as dust-like matter at early stage and as a cosmological constant at later stage. The Chaplygin gas emerges as an effective fluid associated with D-branes and can also be obtained from the Born-Infeld action [11]. Recently, the original Chaplygin gas model was generalized, and subsequently the above equation (16) was modified to the form (known as generalized Chaplygin gas)[12] 


$$
p_{c h}=-\frac{A}{\rho_{c h}^{\alpha}} \quad, \quad 0 \leq \alpha \leq 1
$$

This generalized Chaplygin gas (GCG) model has been studied previously [12]. There are some works on modified Chaplygin gas (MCG) obeying an equation of state [11]

$$
p_{c h}=A \rho_{c h}-\frac{B}{\rho_{c h}^{\alpha}}
$$

and the corresponding evolution of density is

$$
\rho_{c h}=\left[\frac{B}{1+A}+\frac{C}{a^{3(1+\alpha)(1+A)}}\right]^{\frac{1}{1+\alpha}}
$$

where, $A, B$ and $C$ are positive constants. It is obvious from the equation (18) that if $\alpha=1$, then GCG model becomes the original Chaplygin gas model. It can be seen very easily that this energy density interpolates between a dust-like configuration $\left(\rho \simeq \sqrt{B} a^{-3}, p \simeq 0\right)$ in the past and a de-Sitter-like one $(p=-\rho)$ in the late times. This property makes the GCG model an interesting candidate for the unification of dark matter and dark energy. Zhang and Zhu (Ref. [11]) considered an interaction between ordinary Chaplygin gas model and dark matter. Ref [13] has shown that the expression for $H$ obtained from GCG is a good fit for the observational data as far as the background cosmology is concerned.

Since our purpose is to consider interaction between DBI-essence and MCG obeying the equation of state given by equation (19); we consider the conservation equations (14) and (15) involving the interaction term $\delta$. In the present case $\rho_{X} \equiv \rho_{c h}$ and $p_{X} \equiv p_{c h}$. We choose $T=n \dot{\phi}_{D}^{2}$ and $a=t^{m}$ in equations (8) and (11)-(15). We have chosen $T=n \dot{\phi}_{D}^{2}$ because the term $\dot{\phi}_{D}^{2}$ is already there in the expression for $\gamma$ and consequently this choice makes the calculation convenient. Since it is already mentioned that the tension $T$ is a function of the field $\phi_{D}$, the choice seems suitable. Since scale factor $a$ is a function of time $t$, we have chosen it in the power law form as $t^{m}$ with $m>0$ and since it matches the accelerated expansion of the universe, this choice seems suitable. For such choices, we get under interaction, the expression for the density of Chaplygin gas as

$$
\rho_{c h}=\left(\frac{B}{1+A+\delta}+\frac{C}{t^{3 m(1+\alpha)(1+A+\delta)}}\right)^{\frac{1}{1+\alpha}}
$$

Also the expressions of scalar field and the relevant potential for DBI-essence under interaction are obtained as

$$
\begin{aligned}
\phi_{D}= & \int\left[2 \sqrt { \frac { n - 1 } { n } } \left\{\frac{m}{t^{2}}+\frac{1}{2}\left(\frac{B}{1+A+\delta}+\frac{C}{t^{3 m(1+\alpha)(1+A+\delta)}}\right)^{-\frac{\alpha}{1+\alpha}} \times\right.\right. \\
& \left.\left.\left(B-(1+A+\delta)\left(\frac{B}{1+A+\delta}+\frac{C}{t^{3 m(1+\alpha)(1+A+\delta)}}\right)\right)\right\}\right]^{1 / 2} d t
\end{aligned}
$$

and

$$
V_{D}=\frac{3 m^{2}}{t^{2}}-(\gamma-1) n \dot{\phi}_{D}^{2}-\rho_{c h}
$$

Figures 1 and 2 show the variation of $\phi_{D}$ against $t$ and the variation of $V_{D}$ against $\phi_{D}$ respectively in presence of interaction between DBI-essence and MCG. From these figures we see that the DBI scalar field increases with cosmic time $t$, whereas the DBI potential decreases with the field under the interaction. 


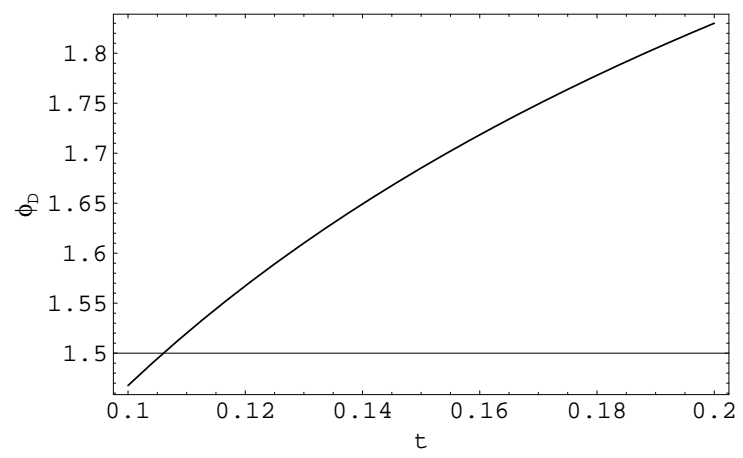

Fig.1

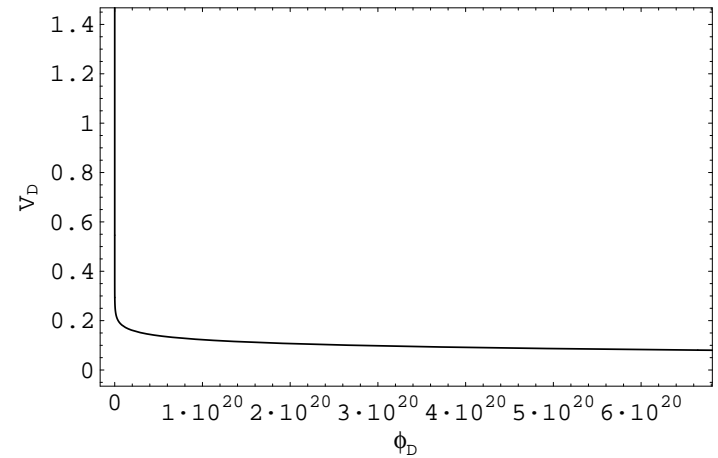

Fig.2

Figs. 1 and 2 show the variation of $\phi_{D}$ against $t$ and the variation of $V_{D}$ against $\phi_{D}$ respectively in presence of interaction between DBI-essence and MCG.

\section{INTERACTION WITH HESSENCE}

The hessence is a kind of simple quintom, which has the Lagrangian density [14]

$$
\mathcal{L}_{D E}=\frac{1}{2}\left[\left(\partial_{\mu} \phi_{1}\right)^{2}-\left(\partial_{\mu} \phi_{2}\right)^{2}\right]-V\left(\phi_{1}, \phi_{2}\right)
$$

where $\phi_{1}$ and $\phi_{2}$ are two real scalar fields and play the roles of quintessence and phantom respectively. Considering a spatially flat Friedmann-Robertson-Walker (FRW) universe and assuming the scalar fields $\phi_{1}$ and $\phi_{2}$ are homogeneous, one obtains the effective equation of state as

$$
\omega=\frac{\dot{\phi}_{1}^{2}-\dot{\phi}_{2}^{2}-2 V\left(\phi_{1}, \phi_{2}\right)}{\dot{\phi}_{1}^{2}-\dot{\phi}_{2}^{2}+2 V\left(\phi_{1}, \phi_{2}\right)}
$$

It is obvious that for $\dot{\phi}_{1}^{2}>\dot{\phi}_{2}^{2}$ we get $\omega>-1$ and for $\dot{\phi}_{1}^{2}<\dot{\phi}_{2}^{2}$ we get $\omega<-1$. In the present work, we consider a quintom model having $V\left(\phi_{1}, \phi_{2}\right)=V\left(\phi_{1}^{2}-\phi_{2}^{2}\right)$. Similar choice of $V$ is available in Wei et al of reference [14]. We consider the action

$$
S=\int d^{4} x \sqrt{-g}\left(-\frac{R}{16 \pi G}+\mathcal{L}_{D E}+\mathcal{L}_{m}\right)
$$

where, $g$ is the determinant of the metric $g_{\mu \nu}, R$ is the Ricci scalar, $\mathcal{L}_{D E}$ and $\mathcal{L}_{m}$ are the Lagrangian densities of dark energy and dark matter respectively. The Lagrangian density of Hessence is given by the equation (23). It can be shown that the Lagrangian remains invariant under the transformation

$$
\begin{aligned}
& \phi_{1} \rightarrow \phi_{1} \cos \alpha-i \phi_{2} \sin \alpha \\
& \phi_{2} \rightarrow-i \phi_{1} \sin \alpha+\phi_{2} \cos \alpha
\end{aligned}
$$

Consequently, the Lagrangian density equation (24) gets the form

$$
\mathcal{L}_{D E}=\frac{1}{2}\left[\left(\partial_{\mu} \phi\right)^{2}-\phi^{2}\left(\partial_{\mu} \theta\right)^{2}\right]-V(\phi)
$$

where, the new variables $(\phi, \theta)$ are given as 

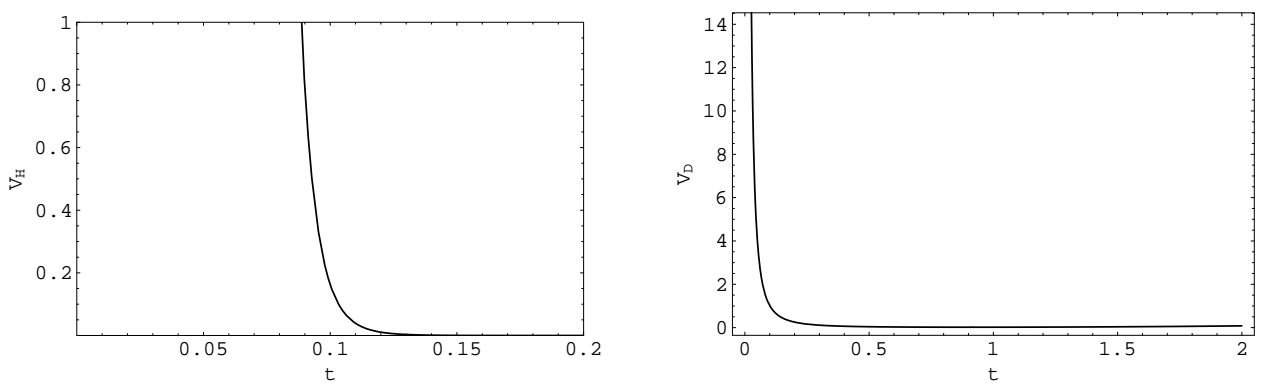

Fig.3

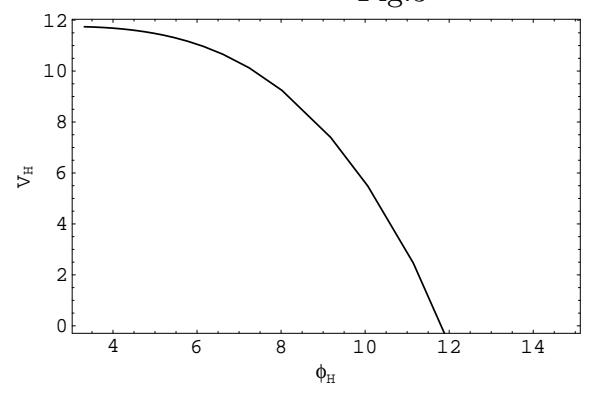

Fig.4

Fig.5
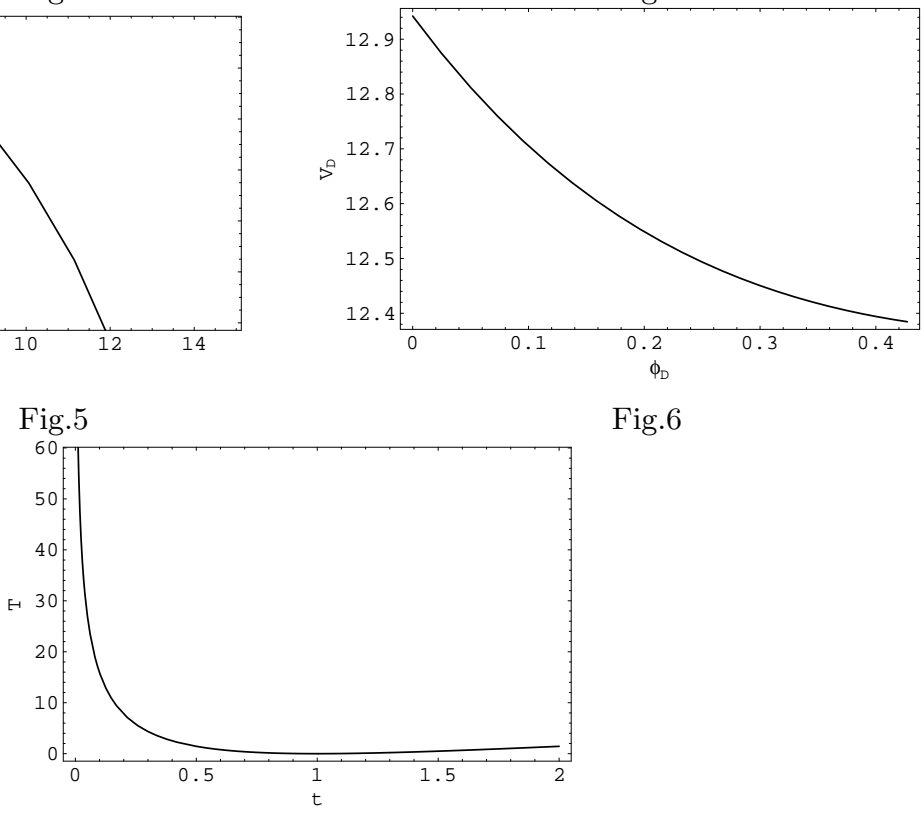

Fig.6

Fig.7

Figs. 3 and 4 show the variation of $V_{H}$ and $V_{D}$ against $t$ in the case of interaction between DBI-essence and hessence. Figs. 5 and 6 show the variations of the above potentials with the corresponding scalar fields in the presence of the said interaction.

Fig. 7 shows the variation of $T\left(\phi_{D}\right)$ with time $t$ in the presence of the said interaction.

$$
\phi_{1}=\phi \cosh \theta \quad ; \quad \phi_{2}=\phi \sinh \theta
$$

From the action given in (26) we get under the assumption of homogeneous $\phi$ and $\theta$ it can be obtained [14]

$$
\begin{gathered}
\ddot{\phi}+3 H \dot{\phi}+\phi \dot{\theta}^{2}+d V / d \phi=0 \\
\phi^{2} \ddot{\theta}+\left(2 \phi \dot{\phi}+3 H \phi^{2}\right) \dot{\theta}=0
\end{gathered}
$$

Detailed derivation of the above equations (30) and (31) is available in Wei et al of reference [14].

Equation (31) implies that 


$$
Q=a^{3} \phi^{2} \dot{\theta}=\text { Constant }
$$

which is associated with the total conserved charge within the physical volume [15]. This relation gives

$$
\dot{\theta}=\frac{Q}{a^{3} \phi^{2}}
$$

From equation (30) one finds that the sign of the conserved charge $\mathrm{Q}$ is determined by the sign of $\dot{\theta}$. The conserved charge $\mathrm{Q}$ is positive for the case $\dot{\theta}>0$ while $\mathrm{Q}$ is negative for the case of $\dot{\theta}<0$.

From equations (30) and (33) we get

$$
\ddot{\phi}+3 H \dot{\phi}+\frac{Q^{2}}{a^{6} \phi^{3}}+\frac{d V}{d \phi}=0
$$

Denoting the scalar field $\phi$ of the hessence by $\phi_{H}$ we get the pressure and density of the hessence as

$$
\begin{aligned}
& \rho_{H}=\frac{1}{2}\left(\dot{\phi}_{H}^{2}-\phi_{H}^{2} \dot{\theta}^{2}\right)+V_{H}\left(\phi_{H}\right) \\
& p_{H}=\frac{1}{2}\left(\dot{\phi}_{H}^{2}-\phi_{H}^{2} \dot{\theta}^{2}\right)-V_{H}\left(\phi_{H}\right)
\end{aligned}
$$

where, $\dot{\theta}=\frac{Q}{a^{3} \phi_{H}^{2}}$.

Now, taking $T\left(\phi_{D}\right)=m \dot{\phi}_{D}^{2}$ and scale factor $a(t)=t^{n}$; we get from the Einstein field equations (3) and (4) that

$$
\frac{n}{t^{2}}=\frac{1}{2}\left(\dot{\phi}_{H}^{2}-\frac{Q^{2}}{t^{6 n} \phi_{H}^{2}}+m \dot{\phi}_{D}^{2}\right)
$$

Choosing $\dot{\phi}_{D}^{2}=\frac{2 n}{m}\left(\frac{1}{t^{2}}\right)$ we get

$$
\begin{gathered}
\phi_{H}=\sqrt{\frac{2 Q}{3 n-1}} t^{1-3 n} \\
\phi_{D}=\sqrt{\frac{2 n}{m}} \ln t
\end{gathered}
$$

Using the conservation equations (14) and (15) with $p_{X} \equiv p_{H}$ and $\rho_{X} \equiv \rho_{H}$ we get, under interaction, the forms of the potentials as

$$
V_{H}=\frac{1}{4} t^{-3 n \delta}\left[(3 n-1) Q t^{3 n(-2+\delta)}\left(\frac{4 \delta}{2-\delta}+\frac{t^{-2+6 n}(2-3 n(2+\delta))}{3 n \delta-2}\right)+C_{1}\right]
$$

and

$$
V_{D}=\frac{2 n^{2}\left(2+3 n \delta+\sqrt{\frac{n}{n-1}}(1-3 n \delta)\right)}{m t^{2}(2+3 n \delta)}+C_{2} t^{3 n \delta}
$$




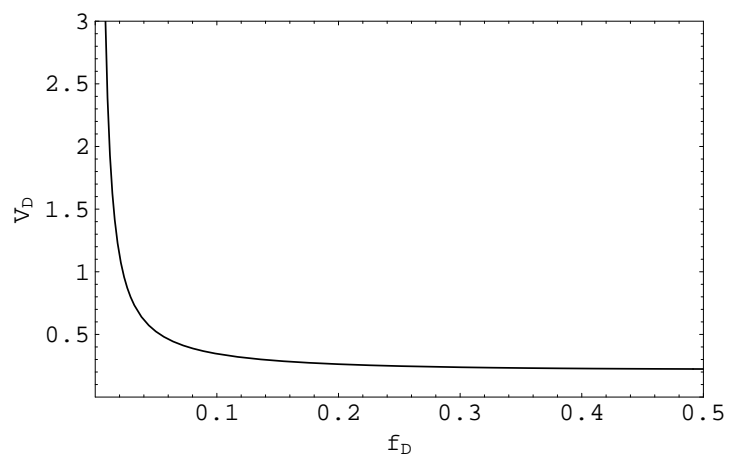

Fig.8

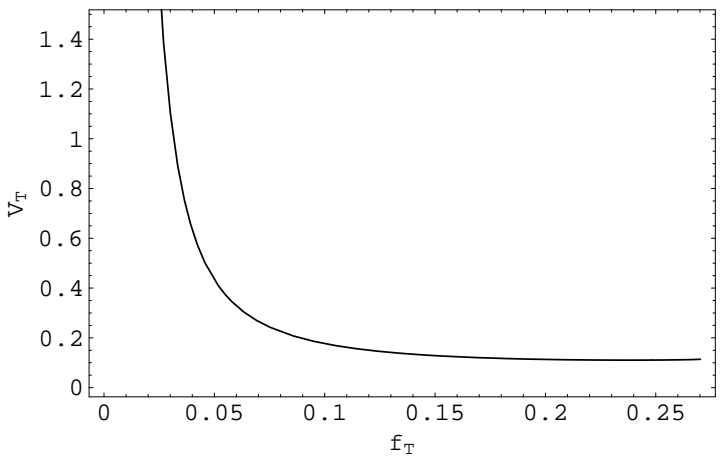

Fig.9

Figs. 8 and 9 show the variation of $V_{D}$ and $V_{T}$ against $\phi_{D}$ and $\phi_{T}$ in the case of interaction between DBI-essence and tachyonic field.
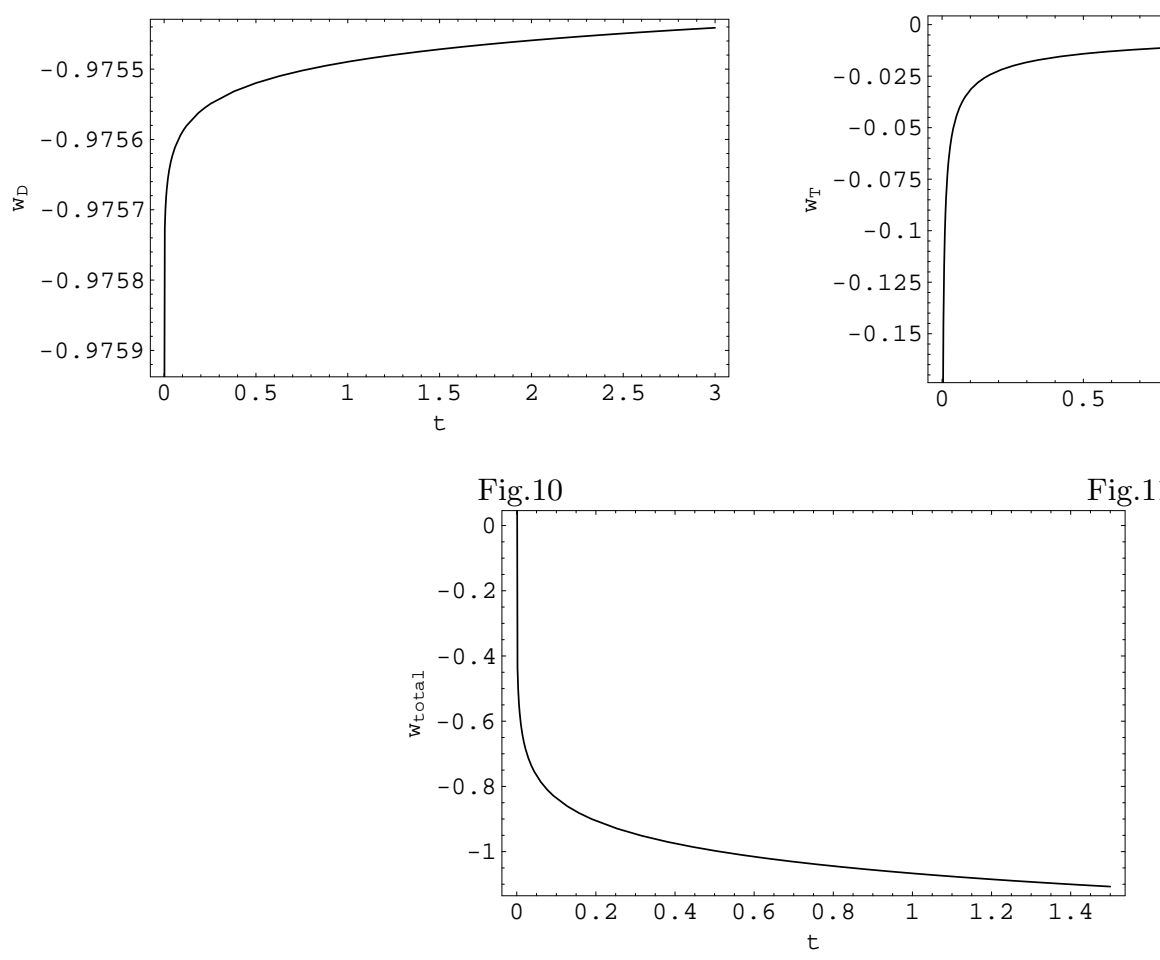

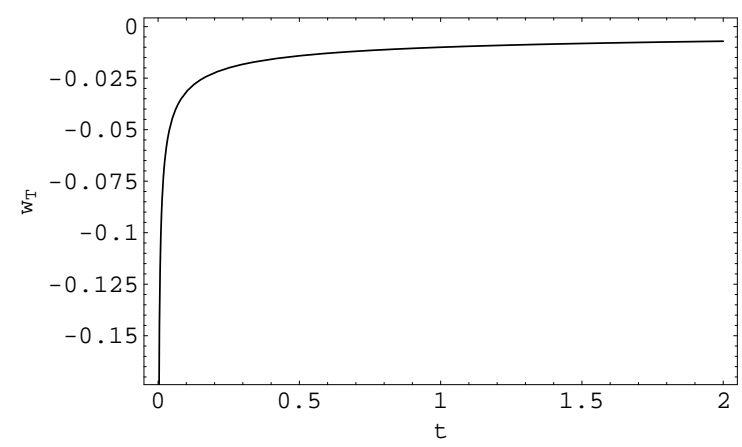

Fig.11

Fig.12 Figs. 10 and 11 show the evolution of $\omega_{D}=\frac{p_{D}}{\rho_{D}}$ and $\omega_{T}=\frac{p_{T}}{\rho_{T}}$ with $t$. in the situation of interaction between tachyonic
field and DBI-essence.

Fig. 12 shows the evolution of $\omega_{\text {total }}=\frac{\left(p_{T}+p_{D}\right)}{\left(\rho_{T}+\rho_{D}\right)}$ with $t$ in the said interaction. 


\section{INTERACTION WITH TACHYONIC FIELD}

The action for the homogeneous tachyon condensate of string theory in a gravitational background is given by $[16]$

$$
S=\int \sqrt{-g} d^{4} x\left[\frac{\mathcal{R}}{16 \pi G}+\mathcal{L}\right]
$$

where, $\mathcal{L}$ is the Lagrangian density given by

$$
\mathcal{L}=-V(\phi) \sqrt{1+g^{\mu \nu} \partial_{\mu} \phi \partial_{\nu} \phi}
$$

where $\phi$ is the tachyonic field, $V(\phi)$ is the tachyonic field potential and $\mathcal{R}$ is the Ricci Scalar. Since we are considering the interacting situation, we would denote the potential for the tachyonic field by $V_{T}$ and the corresponding scalar field by $\phi_{T}$. The energy density $\rho_{T}$ and pressure $p_{T}$ for tachyonic field are given by

$$
\begin{gathered}
\rho_{T}=\frac{V_{T}\left(\phi_{T}\right)}{\sqrt{1-\dot{\phi}_{T}^{2}}} \\
p_{T}=-V_{T}\left(\phi_{T}\right) \sqrt{1-\dot{\phi}_{T}^{2}}
\end{gathered}
$$

In the conservation equations (14) and (15) we consider $\rho_{X} \equiv \rho_{T}$ and $p_{X} \equiv p_{T}$ and assume that $1-\dot{\phi}_{T}^{2}=t^{n}$ and scale factor $a=t^{m}$. Then from the conservation equation (15) we get

$$
V_{T}=e^{\frac{3 n t-m}{m}} t^{-m+3 n(1+\delta)}
$$

Using the above form of tachyonic field potential under interaction in the field equations (8) and (9) with $T=k_{1} \dot{\phi}_{D}^{2}$ and $\dot{\phi}_{D}^{2}=t^{k_{2}}$ we get the form of potential of DBI-essence under interaction with tachyonic field as

$$
V_{D}=\frac{1}{2}\left(\frac{6 n}{t^{2}}+4 k_{1} t^{k_{2}}+\left(3-4 k_{1}\right) \sqrt{\frac{k_{1}}{k_{1}-1}} t^{k_{2}}+\frac{e^{\frac{3 n t^{-m}}{m}} t^{-m+3 n(1+\delta)}\left(3+t^{m}\right)}{\sqrt{t^{m}}}\right)
$$

From equations (11) and (12)we get

$$
\omega_{D}=\frac{\left(k_{1}-\sqrt{k_{1}\left(k_{1}-1\right)}\right) t^{k_{2}}-V_{D}\left(\phi_{D}\right)}{k_{1}\left(\sqrt{\frac{k_{1}}{k_{1}-1}}-1\right) t^{k_{2}}+V_{D}\left(\phi_{D}\right)}
$$

which can be simplified by taking $V_{D}$ from equation (47). Furthermore, from equations (45) and (46) it can be easily obtained that

$$
\omega_{T}=-t^{n}
$$

Evolutions of $\omega_{D}$ and $\omega_{T}$ have been presented in figures 10 and 11 respectively. 


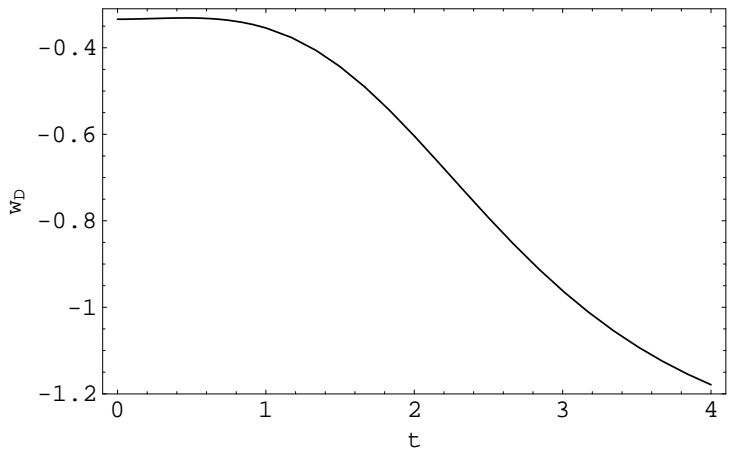

Fig.13

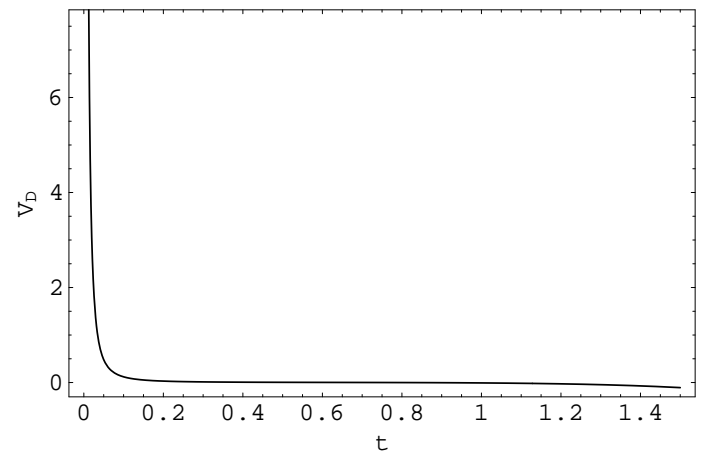

Fig.14

Figs. 13 and 14 show the variation of $\omega_{D}$ and $V_{D}$ against $t$ when DBI-essence is interacting with new agegraphic dark energy.

\section{INTERACTION WITH NEW AGEGRAPHIC DARK ENERGY}

Quantum mechanics together with general relativity leads to the Karolyhazy relation and a corresponding energy density of quantum fluctuations of space-time. Based on this energy density, Cai [17] proposed a dark energy model, the so-called agegraphic dark energy model, in which the age of the universe is introduced as the length measure. The corresponding energy density is given by [17]

$$
\rho_{q}=\frac{3 n^{2} m_{p}^{2}}{T^{2}}
$$

where,

$$
T=\int \frac{d a}{H a}
$$

If we consider a flat FRW universe containing the agegraphic dark energy and pressureless-matter, the the corresponding Friedman equation becomes

$$
H^{2}=\frac{1}{3 m_{p}^{2}}\left(\rho_{m}+\rho_{q}\right)
$$

Introducing the fractional energy densities $\Omega_{i}=\rho_{i} / 3 m_{p}^{2} H^{2}$ we can get

$$
\Omega_{q}=\frac{n^{2}}{H^{2} T^{2}}
$$

and the equation-of-state parameter $\omega_{q}=p_{q} / \rho_{q}$ is given by

$$
\omega_{q}=-1+\frac{2}{3 n} \sqrt{\Omega_{q}}
$$

The agegraphic dark energy was constrained by using some old high redshift objects and type Ia supernovae [18]. A new agegraphic dark energy model was proposed in [19], where the time scale is chosen as the conformal time $\eta$ instead of the age of the universe. For this new agegraphic dark energy, the energy density $\rho_{A}$ is given 


$$
\rho_{A}=\frac{3 n^{2} m_{p}^{2}}{\eta^{2}}
$$

where

$$
\eta=\int \frac{d t}{a}
$$

Thus, $\dot{\eta}=1 / a$. The corresponding fractional energy density is given by

$$
\Omega_{A}=\frac{n^{2}}{H^{2} \eta^{2}}
$$

In the present work, we would consider an interaction between DBI-essence and new agegraphic dark energy. To do so, we consider equations (14) and (15) and in the present case $\rho_{X} \equiv \rho_{A}$ and $p_{X} \equiv p_{A}$. Considering the interaction we obtain the equation-of-state parameter as

$$
\omega_{A}=-1-\delta+\frac{2}{3 n} \frac{\sqrt{\Omega_{A}}}{a}
$$

Now we assume $a=t^{k_{1}}, 1-\dot{\phi}_{D}^{2}=t^{k_{2}}$, and $T=k_{3} \dot{\phi}_{D}^{2}$ and get the potential of DBI-essence under interaction as

$$
V_{D}=\frac{1}{2}\left(\frac{6 k_{1}}{t^{2}}+\left(1-t^{k_{2}}\right)\left(4 k_{3}-\sqrt{\frac{k_{3}}{k_{3}-1}}\left(4 k_{3}-3\right)\right)-\frac{3}{k_{1}}\left(k_{1}-1\right)^{2}\left(-2+3 k_{1}(2+\delta)\right) n^{2} m_{p}^{2} t^{2\left(k_{1}-1\right)}\right)
$$

Using equations (11) and (12), we get the equation of state for DBI-essence under this interaction as

$$
\omega_{D}=\frac{\left(k_{3}-\sqrt{k_{3}\left(k_{3}-1\right)}\right)\left(1-t^{k_{2}}\right)-V_{D}\left(\phi_{D}\right)}{k_{3}\left(\sqrt{\frac{k_{3}}{k_{3}-1}}-1\right)\left(1-t^{k_{2}}\right)+V_{D}\left(\phi_{D}\right)}
$$

which can be simplified by taking $V_{D}$ from equation (59). Evolution of this equation of state parameter $\omega_{D}$ under the given interaction has been presented in figure 13. From this figure, we find that the equation of state parameter is decreasing with time and always negative during evolution.

\section{CONCLUSIONS}

In the present work, we have considered the DBI-essence as a candidate of dark energy interacting with other candidates of dark energies. The other candidates considered here are the modified Chaplygin gas, tachyonic field, hessence and new agegraphic dark energy. We have considered an interaction parameter $\delta$ and accordingly generated the conservation equations in presence of interaction. First we have taken the modified Chaplygin gas as a dark energy interacting with DBI-essence. In this case we have derived the energy density of the modified Chaplygin gas in the presence of interaction, i.e. involving the interaction parameter $\delta$. The scalar field for the DBI-essence has been derived in the presence of interaction and its evolution with time has been viewed in figure 1, which shows that the scalar field is increasing with time. From figure 2, which shows the evolution of the potential of the DBI-essence in this interacting situation, reveals that there is a sharp decrease in the potential with increase in the scalar field. Next we have considered the interaction between the DBI-essence and hessence. In figures 3, 4, and 7 we have seen that the potentials for the DBI-essence and hessence are falling rapidly with increase in time. In figures 5 and 6 , the evolution of potentials are viewed

with increase in the scalar field. In those figures it is revealed that the potentials are falling with increase in 


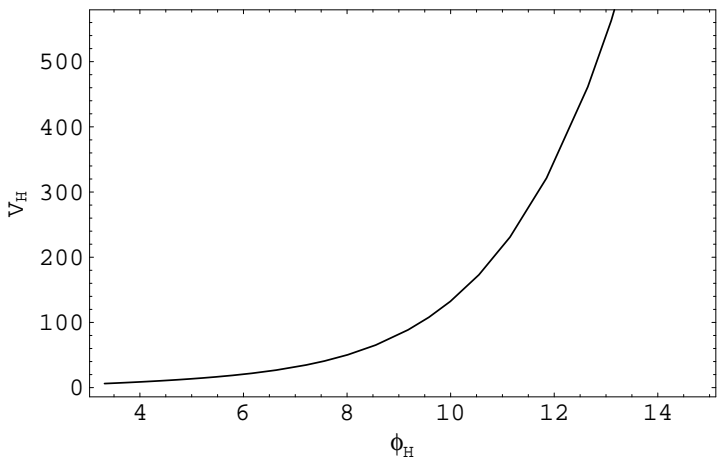

Fig. 15

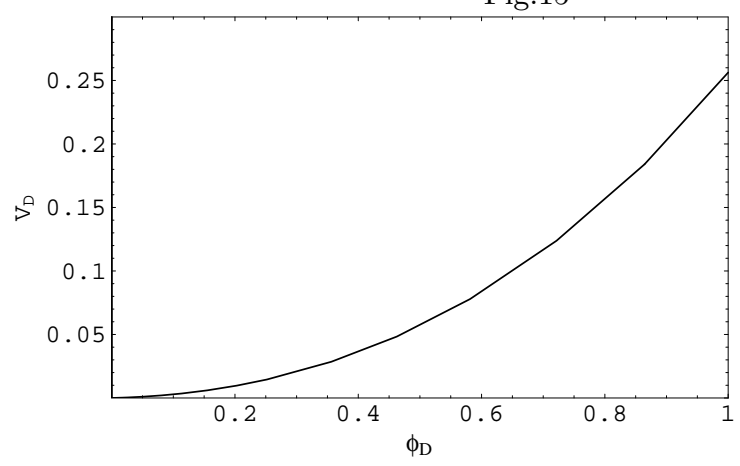

Fig. 17

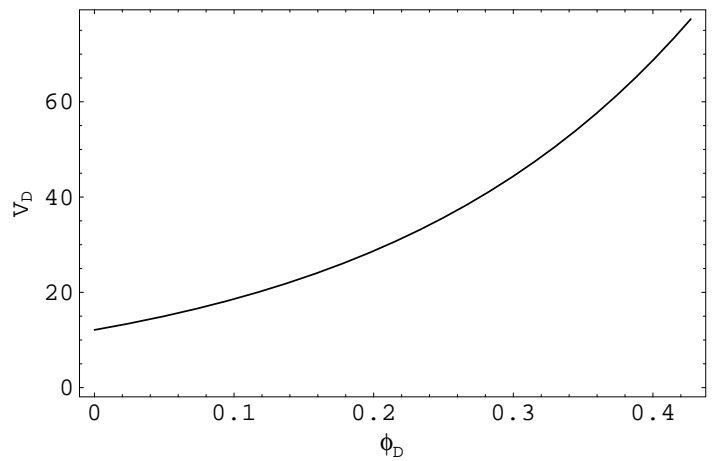

Fig.16

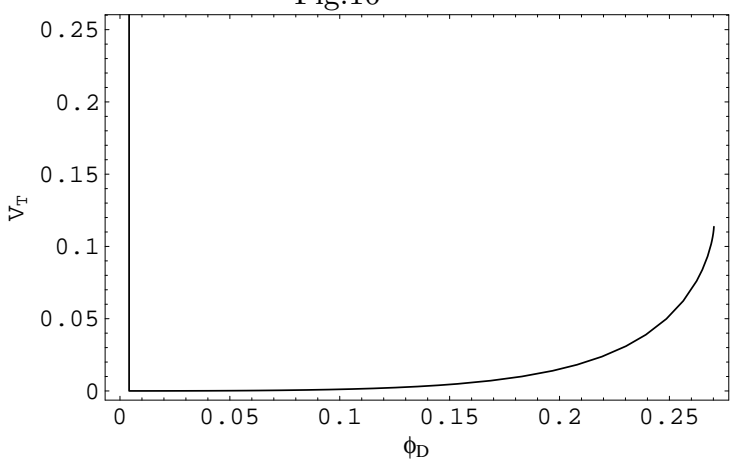

Fig.18

Figs. 15 and 16 show the variation of potential with scalar field when DBI-essence is interacting with hessence with $\delta=0.6$.

Figs. 17 and 18 show the variation of potential with scalar field when DBI-essence is interacting with tachyonic field with $\delta=0.6$.

the scalar fields in the situation of interaction between DBI-essence and hessence. However, the fall is not as sharp as it is in the case of the evolution of the potential with increase in time. The next interaction is between the DBI-essence and tachyonic field. In figures 8 and 9 we have seen that the potentials for both of the dark energy candidates are decreasing with increase in the corresponding scalar fields. In this interaction, we have also considered the equation of the individual as well as the combination of the two interacting dark energies in the figures 10, 11 and 12. In all the cases we find that the equation of state parameters are negative in sign and in the case of combination of the two dark energies it is falling sharply and finally getting stable in the vicinity of -1 . Whereas, in the individual cases, the parameter is increasing with increase in time. Finally, we have considered the interaction between DBI-essence and new agegraphic dark energy. In figure 13, we have plotted the equation of state parameter for DBI-essence in this interacting situation against time $t$. We find in this figure that the said parameter is gradually decreasing with time and in lying approximately between -1.2 and 0 . The potential in this case is showing the same sharply falling pattern with increase in $t$ as it was in the previous interactions. It is, therefore, observed from the interactions that the potentials are always experiencing sharp fall with increase in time in all the four cases. However, the fall in the potential is not so sharp when it is considered against the corresponding scalar field. It should be noted that in all the cases we have taken the interaction parameter $\delta$ very close to 0 . Similar nature in the figures have been observed in the case when the interaction parameter is negative. Now we examine the $V(\phi)-\phi$ relations if the interaction term is taken at larger value. In the case of interaction with modified Chaplygin gas, $\delta$ was taken equal to 0.005. Taking larger values we find that the similar nature as it was earlier is available. In the case of interaction with hessence, we had taken $\delta=0.006$. Now we take $\delta=0.6$, and we find that the potentials are increasing with increase in the scalar fields (figures 15 and 16). Now we consider the interaction with tachyon. Earlier we had taken $\delta=0.05$. Now, taking $\delta=0.6$ we find that the potential $V$ is increasing with increase in the scalar field $\phi$ (figures 17 and 18). 


\section{Acknowledgement:}

The authors are thankful to IUCAA, Pune, India for warm hospitality where part of the work was carried out. Also UD is thankful to UGC, Govt. of India for providing research project grant (No. 32-157/2006(SR)).

\section{References:}

[1] E. J. Copeland, M. Sami, and S. Tsujikwa, Int. J. Mod. Phys. D 151753 (2006); S. Perlmutter et al., Astrophys. J. 517565 (1999).

[2] A. G. Riess et al., Astrophys. J. 1161009 (1998); A. G. Riess et al., Astron. J. 117707 (1999); S. Boughn and R. Crittenden, Nature 42745 (2004)

[3] J. Martin and M. Yamaguchi, Phys. Rev. D 77123508 (2008); L. P. Chimento , R. Lazkoz, and I. Sendra, Gen. Relativ. Gravit. DOI:10.1007/s10714-009-0901-z (2009).

[4] S. Weinberg, Rev. Mod. Phys. 611 (1989); D. N. Spergel et al. arXiv:astro-ph/0603449.

[5] L. P. Chimento, R. Lazkoz, and I. Sendra, arXiv:0904.1114v2].

[6] S. Kachru et al., JCAP 10013 (2003); E. Silverstein and D. Tong, Phys. Rev. D 70103505 (2004).

[7] B. Gumjudpai and J. Ward, Phys. Rev. D 80023528 (2009); J. Martin and M. Yamaguchi, Phys. Rev. D 77123508 (2008).

[8] J. E. Lidsey and I. Huston, JCAP 0707002 (2007); D. Baumann and L. McAllister, Phys. Rev. D 75 123508 (2007).

[9] M. S. Berger, H. Shojaei, Phys. Rev. D 74043530 (2006).

[10] R. Herrera, D. Pavon and W. Zimdahl, Gen. Rel. Grav. 362161 (2004); R. G. Cai and A. Wang, JCAP 0503002 (2005); Z. K. Guo, R. G. Cai and Y. Z. Zhang, JCAP 0505002 (2005); T. Gonzalez and I. Quiros, [gr-qc/0707.2089].

[11] H. Zhang and Z. Zhu, Phys. Rev. D 73043518 (2006); U. Debnath, Astrophys. Space Sci. 312295 (2007).

[12] U. Alam, V. Sahni, T. D. Saini and A. A. Starobinsky, Mon. Not. R. Astron. Soc. 3441057 (2003); V. Gorini, A. Kamenshchik and U. Moschella, Phys. Rev. D 67063509 (2003); M. C. Bento, O. Bertolami and A. A. Sen, Phys. Rev. D 66043507 (2002).

[13] T. Barreiro and A. A. Sen, Phys. Rev. D 70124013 (2004).

[14] H. Wei, R. G. Cai and D. F. Zeng, Class. Quantum Grav. 223189 (2005); Z. K. Guo, Y. S. Piao, X. M. Zhang and Y. Z. Zhang, Phys. Lett. B 608177 (2005); X. F. Zhang, H. Li, Y. S. Piao and X. M. Zhang, Mod. Phys. Lett. A 21231 (2006); H. Wei, N. Tang, and S. N. Zhang, Phys. Rev. D 75 043009 (2007),W. Zhao, Phys. Lett. B 65597 (2007).

[15] H. Wei, R. G. Cai, Phys. Rev. D 72123507 (2005) ; M. Alimohammadi, H. M. Sadjadi, Phys. Rev. D 73 083527 (2006); H. Wei, N. Tang and S. N. Zhang, Phys. Rev. D 75043009 (2007).

[16] S. Chattopadhyay, U. Debnath and G. Chattopadhyay, Astrophys. Space Sci 31441 (2008).

[17] R. G. Cai, Phys. Lett. B 657228 (2007); J. Zhang, X. Zhang and H. Liu, Eur. Phys. J. C.54 303 (2008). [18]H. Wei and R. G. Cai, Phys. Lett. B 663 1(2008); Y. Zhang, H. Li, X. Wu, H. Wei and R. G. Cai, arXiv:0708.1214.

[19] H. Wei and R. G. Cai, Phys. Lett. B 660113 (2008). 